For the cycloid

$$
\frac{1}{\rho^{2}}=\frac{1}{16 a^{2} \sin ^{2} \frac{1}{2} \bar{\theta}}=\frac{1}{8 a\left(s-\frac{s^{2}}{8 a}\right)}=\frac{1}{8 a y},
$$

and after substitution of these values in $\bar{K}_{0}$ we find

$$
\bar{K}_{0}=\frac{1}{8 a y}\left\{2 a\left(y^{\prime 2}-3\right)+y\right\}
$$

or making use of the extremal equation,

$$
\bar{K}_{0}=-\frac{1}{2} y, \text { i. e., } \quad \bar{K}_{0}<0 .
$$

By means of theorem $A$, we have the result:

For the brachistochrone problem there is no conjugate point to any point $P$ lying on the same cycloid arch with $P$.

The University of Wisconsin.

\title{
A SIMPLER PROOF OF LIE'S THEOREM FOR ORDINARY DIFFERENTIAL EQUATIONS.
}

BY PROFEssor L. D. AMES.

(Read before the Chicago Section of the American Mathematical Society, April 9, 1909.)

The following theorem is essentially equivalent to Lie's principal theorem concerning the integration of the differential equation $\Omega\left(x, y, y^{\prime}\right)=0$ when it is invariant under a known group. As stated here, this theorem makes no use of the idea of a group.

Theorem. Given any differential equation of the form

$$
\Omega\left(x, y, y^{\prime}\right)=0
$$

which can be solved in the form

$$
X(x, y) y^{\prime}-Y(x, y)=0 ;
$$

if $\xi(x, y)$ and $\eta(x, y)$ are such functions that

$$
X \eta-Y \xi \neq 0,
$$


1909.] SIMPLER PROOF OF IIE'S THEOREM.

then a necessary and sufficient condition that

be exact is that

$$
\frac{X}{X \eta-Y \xi} Y^{\prime}-\frac{Y}{X \eta-Y \xi}=0
$$

(4) $\xi \frac{\partial \Omega}{\partial x}+\eta \frac{\partial \Omega}{\partial y}+\left(\frac{\partial \eta}{\partial x}+y^{\prime} \frac{\partial \eta}{\partial y}-y^{\prime} \frac{\partial \xi}{\partial x}-y^{\prime 2} \frac{\partial \xi}{\partial y}\right) \frac{\partial \Omega}{\partial y^{\prime}}=0 .^{*}$

Proof. Let us first assume that the given equation is in the solved form

$$
\Omega_{1}\left(x, y, y^{\prime}\right) \equiv X(x, y) y^{\prime}-Y(x, y) .
$$

A necessary and sufficient condition that (3) be exact is that

$$
\frac{\partial}{\partial x}\left(\frac{X}{X \eta-Y \xi}\right)+\frac{\partial}{\partial y}\left(\frac{Y}{X \eta-Y \xi}\right)=0 .
$$

If we expand (6), drop the denominator, collect, change signs, divide by $X$, and put $Y / X=y^{\prime}$ from (2), we obtain the required condition (4). All these steps are possible since $X \eta-Y \xi \neq 0$ by hypothesis, and $X \neq 0$.

Next let the given equation be any equation of the form

$$
\Omega\left(x, y, y^{\prime}\right)=0
$$

which can be solved in the form

$$
\Omega_{1}\left(x, y, y^{\prime}\right) \equiv X y^{\prime}-Y=0
$$

throughout a region $R$ in the three variables $x, y, y^{\prime}$, thought of as coordinates of a point in three dimensions. Differentiating each, we obtain

It follows that

$$
\begin{gathered}
\frac{\partial \Omega}{\partial x} d x+\frac{\partial \Omega}{\partial y} d y+\frac{\partial \Omega}{\partial y^{\prime}} d y^{\prime}=0 \\
\frac{\partial \Omega_{1}}{\partial x} d x+\frac{\partial \Omega_{1}}{\partial y} d y+\frac{\partial \Omega_{1}}{\partial y^{\prime}} d y^{\prime}=0 .
\end{gathered}
$$

$$
\frac{\frac{\partial \Omega}{\partial x}}{\frac{\partial \Omega_{1}}{\partial x}}=\frac{\frac{\partial \Omega}{\partial y}}{\frac{\partial \Omega_{1}}{\partial y}}=\frac{\frac{\partial \Omega}{\partial z}}{\frac{\partial \Omega_{1}}{\partial z}}
$$

* In the language of the Lie theory this is the condition that $\Omega\left(x, y, y^{\prime}\right)=0$ be invariant under the group $U f=\xi(\partial f / \partial x)+\eta(\partial f / \partial y)$. See Lie-Scheffers, Vorlesungen über Differentialgleichungen mit bekannten infinitesimalen Transformationen, p. 97. 
Hence the results obtained by using $\Omega$ and $\Omega_{1}$ respectively in (4) differ only by a factor, and the condition (4) applies equally well to the equation in the general form. This proves the theorem as stated.

The Lie theory gives no general method of finding the group of a given differential equation, and the problem of finding such a group is precisely the problem of finding $\xi$ and $\eta$, solutions of $(4)$.

If $\xi$ and $\eta$ are chosen in advance, the general form of the differential equation which can be integrated by their aid can be determined in precisely the same manner as in the Lie theory. Thus the Lie theory offers no formal aid in the solution of the above mentioned type of equation not furnished by this theorem.

However, there are some interesting considerations of a geometrical character due to Monge, Lie, and others which are suggestive occasionally; it is possible to restate some of these also, and the author proposes to do this in a later paper.

The method used in this paper can be applied to other types of differential equations, and Lie's essential results for those types obtained. These types will form the subject matter of another paper.

UNIVERSTTY OF MISSOURI, March 3, 1909.

\section{HEATH'S EUCLID.}

The Thirteen Books of Euclid's Elements Translated from the Text of Heiberg with Introduction and Commentary. By T. L. Heath, C.B., Sc.D. Cambridge, University Press, 1908. 8 vo. 3 volumes. \$13.50.

IT may properly be said of Dr. Heath's latest contribution to mathematical literature - and may it not be his last! - that it is characteristically British, and perhaps nothing better could be said of a work on the great mathematical classic of the world. We are tempted to think that England does not produce the great works of Germany, say in the history of mathematics as also in the theory of this science, and we point to Cantor and to Hankel, to Braunmühl and possibly to Günther, or even to Simon, as justifying this thought. And yet, when the English scholar does bring out a book, it is quite as when a British general takes possession of a conquered province - there is 